\title{
GLOBESAR-2: O PROGRAMA DE AVALIAÇÃO DE DADOS DO RADARSAT NAS GEOCIÊNCIAS
}

\author{
WALDIR RENATO PARADELLA ${ }^{+*}$, THELMA KRUG*, ROBERT LANDRY **, RON W. PIETSCH***
}

\begin{abstract}
The RADARSAT-1, launched in November 1995, is the first operatipnally oriented Earth observation satellite to carry a SAR (Synthetic Aperture Radar) imaging sensor. A key capability that distinguishes RADARSAT from other satellite SAR systems (ERS-1, ERS-2, JERS-1) is the flexibility it offers in terms of image acquisition modes. The system is the first commercial radar satellite to use a "steerable" antenna which enables terrestrial observation from selected incidences angles $\left(10^{\circ}\right.$ to $\left.59^{\circ}\right)$. RADARSAT has 35 distinct beam and incidence angle positions with spatial resolution varying from $10-100 \mathrm{~m}$. One advantage of controlling the variation of incidence angle of the SAR image is that the user is able to acquire two images of the same area (from distinct orbital passes) at different incidence angles, and the two scenes can be viewed together using traditional stereoscopic methods (three-dimensional perspective of the terrain). RADARSAT-1 is the first of a three-satellite program. Efforts have already begun aiming at the specification of RADARSAT-2 which will have an anticipated launch around the year 2000. A third satellite is envisaged to continue the RADARSAT program to the year 2010 and beyond. GlobeSAR-2 is a technology transfer, training, and applications development program, based on RADARSAT-1 data, carried out in 11 countries in Latin America. The program is led by the Canada Centre for Remote Sensing (CCRS) with the South American portion of the program funded by the Canadian International Development Agency (CIDA) and International Development Research Centre (IDRC). RADARSAT International Inc. (RSI), PCI, and Atlantis Scientific are private sectors partners in GlobeSAR-2. CIDA funding of $\$ 4.3$ million (CDN) supports eight countries and three countries are supported by funding from IDRC. Expected expenditures in Brazil for the GlobeSAR-2 Program are estimated approximately at $\$ 500,000$ (CDN) covering the purchase of image processing software (PCI and Atlantis Scientific) and RADARSAT images (RSI), training, in-country travel for GlobeSAR-2 participants to field-work, workshops, national seminars, and national and international symposia. In addition, University training materiais developed by the program will be widely distributed to Brazilian universities. The GlobeSAR-2 Program in Brazil is coordinated by INPE. The GlobeSAR-2 program goals are: (1) to learn how to use satellite SAR data, particularly RADARSAT-1 data, for geosciences applications in distinct environments; $(2)$ to enhance the resource management capabilities in the participating countries through demonstration and training of SAR remote sensing, in particular RADARSAT-1, and (3) to demonstrate the value of RADARSAT-1 applications for improved natural resource and environmental monitoring and planning, promote the integration of RADARSAT-1 information into each country's ongoing programs and projects, and help to establish and enhance the radar remote sensing capacity in each of the participating countries, in particular the integration of RADARSAT with other data. A baseline program for GlobeS AR-2 in Brazil was defined in Feb 95 based on collaborative projects involving Brazilian researches mainly from Universities and national agencies. A call for experiments was issued to Brazilian researches in March 96 and 28 proposals were received at INPE. The proposals were evaluated and awarded by a CCRS/RSI team on a competitive basis. The final decisions concerning the acceptance of the proposals were made by the GlobeSAR-2 Sponsors in October 96, and 10 proposals were approved to receive RADARSAT scenes and 14 proposals were selected to received a SAR educational/training package and also radar analysis software licenses. This paper briefly presents the GlobeSAR-2 program, highlights the educational and training approach being implemented, and also provides an overview of the applications selected for the Brazilian component of the program.
\end{abstract}

INTRODUÇÃO E CONTEXTO A aquisição da primeira imagem do RADARSAT-1 em 28 de Novembro de 1995 , completou o ciclo de sistemas orbitais com radares imageadores previsto para a década de noventa. Atualmente, imagens orbitais baseadas em SAR (Synthetic Aperture Radar) estão disponíveis através dos Programas ERS-1 e ERS-2 (Comunidade Europeia), JERS-1 (Japão) e RADARSAT-1 (Canadá). Em contraste com esta grande disponibilidade de dados, ainda é muita restrita a cultura no país na extração de informações com estes sensores. Discussões sobre a interação energia/ matéria no espectro das microondas, sobre as novas características do imageamento, dos parâmetros dos sistemas e dos alvos que influenciam nas respostas detectadas, sobre o processamentos digital específico no tratamento destes dados, são escassas na literatura disponível aos usuários potenciais. Maiores detalhes sobre as características de imageamento com radar podem ser vistos em Paradella (1996a). Aspectos gerais do RADARSAT podem ser vistos em Paradella (1996b). Ângulo de incidência, resolução espacial e largura da faixa imageada não fixos, representam a maior diferença entre o RADARSAT-1 e os sistemas anteriores (Figura 1, Tabela 1). Estas características inovadoras deste sistema estimularam a implementação do GlobeSAR-2, um programa de avaliação do RADARSAT-1 em aplicações nas Geociências, com componentes de geração de conhecimento (pesquisa), formação de recursos humanos especializados (treinamento) e transferência de tecnologia aos usuários da América Latina.

O GlobeSAR-2 foi concebido no sucesso do primeiro grande experimento com radar imageador em aeronave, operando na banda $\mathrm{C}$, que foi desenvolvido na América do Sul, particularmente na Região Amazônica, o SAREX' 92 (South American Radar Experiment). O SAREX' 92 foi um experimento conduzido pela ES A (European Space Agency) e pelo CCRS (Canada Centre for Remote Sensing), com suporte da CIDA (Canadian International Development Agency), para a aquisição de dados de radar por aeronave de regiões de florestas tropicais úmidas do Brasil, Costa Rica, Colômbia, Guiana, Guiana Francesa e Venezuela. A campanha ocorreu em Abril de 1992, quando foram coletados dados em uma extensão de cerca de $6.000 \mathrm{Km}$. O principal objetivo do SAREX foi adquirir imagens de radar imageador na banda $\mathrm{C}$ $(5,6 \mathrm{~cm})$, sob diferentes polarizações e geometria de visada. Este acervo de dados serviu de base para gerar conhecimento na extração de informações de alvos tropicais com radares aeroportados e orbitais (ERS-1, ERS-2 e RADARSAT-1). Como plataforma foi usado o Convair 580 do CCRS, que operou a $6 \mathrm{Km}$ de altitude, com um SAR na banda C, com polarizações $\mathrm{HH}, \mathrm{HV}$ e $\mathrm{VV}$, e imageamentos em várias geometrias de visadas. No Brasil, foram selecionadas áreas na Amazónia com diversidades físiográficas e distintas apli-

+ Coordenador Nacional do GlobeSAR-2

* Instituto Nacional de Pesquisas Espaciais (INPE) - 12227-010 - São José dos Campos, SP - Brasil, waldir@ltid.inpe.br, thelma@ltid.inpe.br

** Canada Centre for Remote Sensing (CCRS) - Kl A OY7 - Ottawa, ON - Canadá - Robert.Landry@geocan.nrcan.gc.ca

*** Dendron Resources Surveys Inc. - K1Z 5L9 - Ottawa - ON - Canadá - Ron.Pietsch@geocan.nrcan.gc.ca 


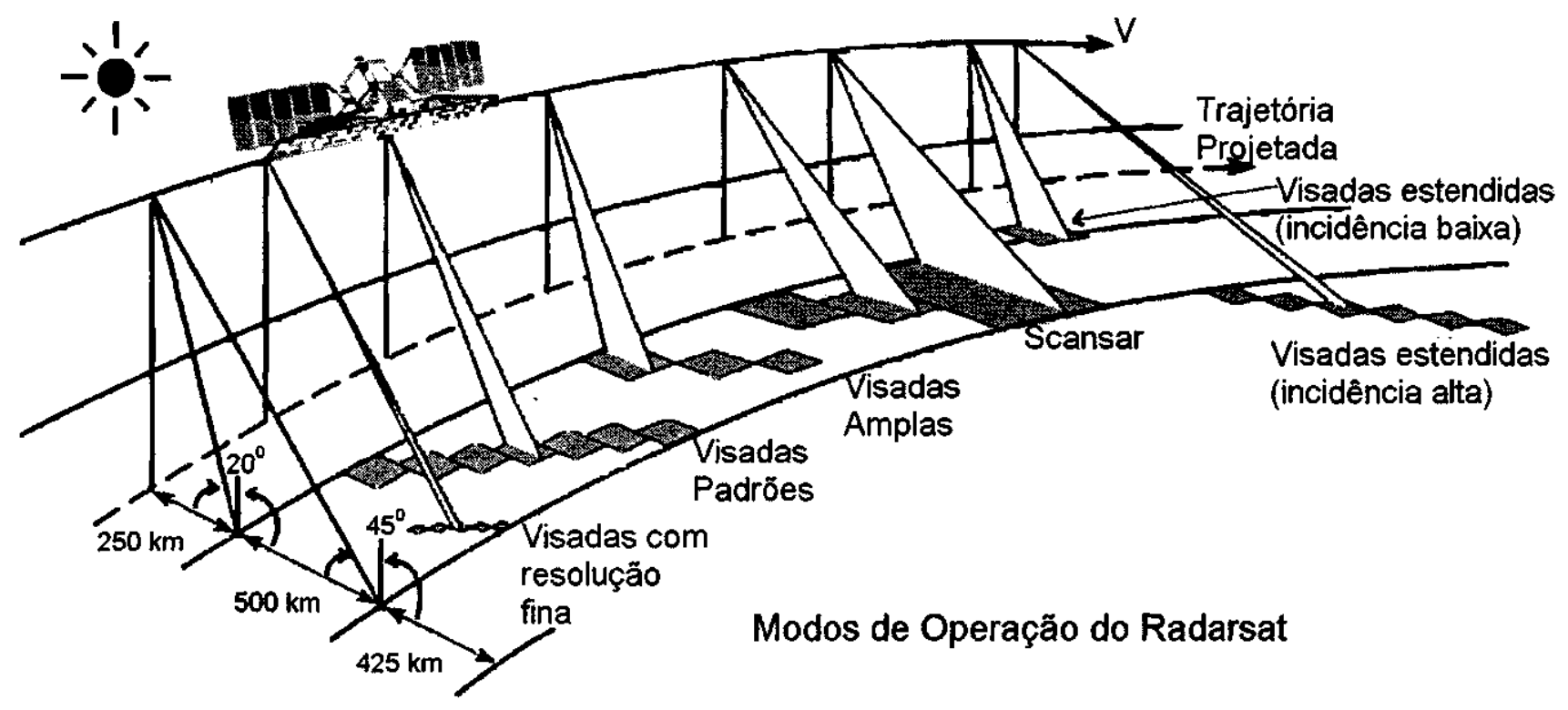

Figura 1. Modos de Operação do RADARSAT-1

Tabela 1. Especificações do RADARSAT-1

\begin{tabular}{|c|c|c|c|c|c|c|}
\hline $\begin{array}{l}\text { Moda de } \\
\text { Vlsenda }\end{array}$ & $\begin{array}{l}\text { Posleario } \\
\text { do Fetixt }\end{array}$ & $\begin{array}{l}\text { Angulo de } \\
\text { Incldencila } \\
\text { (graus) }\end{array}$ & $\begin{array}{c}\text { Resul } \\
\text { Kunge }\end{array}$ & $\begin{array}{l}\text { Aucinio } \\
\text { Aximute (m) }\end{array}$ & $\underset{\substack{\text { Areas } \\
\text { Numinal } \\
(\mathbf{K} m)}}{ }$ & $\begin{array}{l}\text { Némern } \\
\text { de } \\
\text { Lools } \\
\text { Efietivo }\end{array}$ \\
\hline $\begin{array}{c}\text { Resoluçăd } \\
\text { Fins } \\
\text { (Fine) }\end{array}$ & $\begin{array}{l}11 \\
172 \\
173 \\
174 \\
155\end{array}$ & $\begin{array}{l}37-40 \\
39-42 \\
41-44 \\
43-46 \\
45.47\end{array}$ & $\begin{array}{l}8,3 \\
7,9 \\
7,6 \\
7,3 \\
7,1\end{array}$ & $\begin{array}{l}8,4 \\
8.4 \\
8,4 \\
8,4 \\
8,4\end{array}$ & $\begin{array}{l}50 \times 50 \\
50 \times 50 \\
50 \times 50 \\
50 \times 50 \\
50 \times 50\end{array}$ & $\begin{array}{l}1 \\
1 \\
1 \\
1\end{array}$ \\
\hline $\begin{array}{c}\text { Pudrín } \\
\text { (Standard) }\end{array}$ & $\begin{array}{l}51 \\
52 \\
53 \\
54 \\
55 \\
56 \\
57 \\
\end{array}$ & $\begin{array}{l}29-27 \\
24+31 \\
30-37 \\
34-40 \\
36-42 \\
41-46 \\
45-49\end{array}$ & $\begin{array}{l}24,2 \\
20,4 \\
25,3 \\
24,4 \\
24,1 \\
20,3 \\
19,1 \\
\end{array}$ & $\begin{array}{l}27,0 \\
27,0 \\
27,0 \\
27,0 \\
27,0 \\
27,0 \\
27,0 \\
\end{array}$ & $\begin{array}{l}100 \times 100 \\
100 \times 100 \\
160 \times 100 \\
100 \times 100 \\
100 \times 100 \\
100 \times 100 \\
100 \times 100\end{array}$ & $\begin{array}{l}4 \\
4 \\
4 \\
4 \\
4\end{array}$ \\
\hline $\begin{array}{l}\text { Larga } \\
\text { (wide) }\end{array}$ & $\begin{array}{l}\begin{array}{l}W_{1} \\
\text { W2 } \\
\text { w3 }_{3}\end{array} \\
\end{array}$ & $\begin{array}{l}29-31 \\
31 \cdot 39 \\
39-45 \\
\end{array}$ & $\begin{array}{l}33,8 \\
24,6 \\
2,1,3 \\
\end{array}$ & $\begin{array}{l}27,0 \\
27,0 \\
27,0\end{array}$ & $\begin{array}{l}150 \times 150 \\
150 \times 150 \\
150 \times 150 \\
150 \times 150\end{array}$ & $\begin{array}{l}3,1 \\
3,1 \\
3,1 \\
\end{array}$ \\
\hline $\begin{array}{c}\text { ScanSAR } \\
\text { (Clistreets } A \text { \& B })\end{array}$ & $\begin{array}{l}\text { SCNA } \\
\text { SCNAB }\end{array}$ & $\begin{array}{l}30.39 \\
31.46\end{array}$ & $\begin{array}{l}50^{\circ} \\
500^{\circ}\end{array}$ & $\begin{array}{l}50^{*} \\
\text { 50* }\end{array}$ & $\begin{array}{l}300 \times 3000 \\
3100 \times 3400\end{array}$ & $\begin{array}{l}3,5 \\
3.5\end{array}$ \\
\hline $\begin{array}{c}\text { ScanSAR } \\
\text { (LnISD) }\end{array}$ & scw & $20-49$ & 190* & 100* & $\operatorname{sen} \times 500$ & 7 \\
\hline $\begin{array}{l}\text { Bstendido } \\
\text { (Allo ingertios) }\end{array}$ & $\begin{array}{l}\text { EH1 } \\
\text { EH2 } \\
\text { EH3 } \\
\text { EH4 } \\
\text { EHS } \\
\text { EH6 }\end{array}$ & $\begin{array}{l}49-52 \\
50-53 \\
52-55 \\
54.57 \\
56-58 \\
57.59\end{array}$ & $\begin{array}{l}18,0 \\
17,7 \\
17,5 \\
16,8 \\
16,6 \\
16,4\end{array}$ & $\begin{array}{l}27,0 \\
27,0 \\
27,0 \\
27,0 \\
27,0 \\
27,6\end{array}$ & $\begin{array}{l}75 \times 75 \\
75 \times 75 \\
75 \times 75 \\
75 \times 75 \\
75 \times 75 \\
75 \times 75\end{array}$ & $\begin{array}{l}, 1 \\
3,1 \\
3,1 \\
3,1 \\
3,1 \\
3,1 \\
\end{array}$ \\
\hline $\begin{array}{c}\text { Estendida } \\
\text { (Balxo Amguto) }\end{array}$ & EL1 & 10.23 & 39.1 & 27,0 & $170 \times 170$ & 3,1 \\
\hline
\end{tabular}

Resolução varia com o range.

cações: Carajás-PA (mapeamento geológico, prospecção mineral, geobotânica), Tucuruí-PA (estudos de impacto ambiental de grande reservatório), Lago Grande de Monte Alegre-PA (monitoramento de planície de inundação), Floresta Nacional de Tapajós-PA (inventário florestal) e Rio Branco e Sena Madureira/AC (uso da terra, geomorfologia). A pesquisa no Brasil teve a coordenação do INPE e participação do CCRS, DOCEGEO, CPRM, Ibama, Eletronorte e Funtac. Detalhes e resultados sobre o SAREX podem ser vistos no Simpósio da ESA sobre o experimento (ESA 1993). O SAREX teve a continuidade no Brasil com o nome de ProRadar, com término em Março de 1998.

Quase simultaneamente ao SAREX' 92, um novo programa de 3 anos de duração, o GlobeSAR-1, teve início no CCRS em 1993. Em essência, este programa, com fundos das agências do Canada, CIDA e do IDRC (International Development Research Centre), constou da aquisição de dados do
Convair 580 em áreas testes na China, Jordânia, Quénia, Malásia, Marrocos, Tanzânia, Tailândia, Tunísia, Uganda, Vietname, Indonésia e Israel, envolvendo várias aplicações no contexto de simulação de dados do RADARSAT-1. Embora dados orbitais do ERS-1 e JERS-1 tenham sido muito utilizados nestes experimentos, a grande ênfase do SAREX, ProRADAR e GlobeSAR-1 esteve dirigida para a extração de informações com imagens aeroportadas na banda C-HH, simulando o RADARSAT-1. Uma revisão das aplicações estudadas durante o GlobeSAR-1 pode ser vista em Brown et al. (1996).

Como consequência natural dos programas anteriores, $o$ advento do RADARSAT-1 favoreceu a que um programa similar, agora baseado em dados orbitais, pudesse ser implementado. O GlobeSAR-2 está restrito a América Latina, com a participação do Brasil, Argentina, Bolívia, Chile, Colômbia, Costa Rica, Honduras, Panamá, Peru, Uruguai e Venezuela. Um total aproximado de 4.3 milhões de dólares canadenses foi alocado pela CIDA para o desenvolvimento do programa em oito países (incluindo o Brasil) e três países restantes recebem suporte financeiro independente do IDRC. Os recursos diretos do GlobeSAR-2 para o Brasil são estimados em aproximadamente meio milhão de dólares canadenses, cobrindo a aquisição de softwares para processamento de imagens de sensores remotos, particularmente de radar (PCI e Atlantis Scientific), imagens do RADARSAT-1, treinamento de pessoal, despesas no país para trabalhos de campo, participação de seminários e workshops nacionais e internacionais. Em adição, material de treinamento desenvolvido pelo Programa (imagens digitais, apostilas, transparências, etc.) será distribuído aos docentes das universidades participantes do Programa, bem como, um programa de intercâmbio entre docentes e alunos de universidades brasileiras e canadenses está sendo implementado. Concebido para três anos de duração, desde o seu início em 1996 até o presente, 80 projetos foram aprovados nestes 11 países. De modo geral, o programa tem como objetivo maior criar capacitação nacional no planejamento, levantamento e monitoramento de recursos naturais da América Latina, tendo com fonte básica de informação, dados de sensoriamento remoto fornecidos pelo RADAR- 
SAT-1. Este objetivo será alcançado através da componente educacional do programa, com o treinamento dos participantes em análise, interpretação e uso de dados do RADARS AT1 , adquiridos em condições adequadas à cada tipo de aplicações dos projetos aprovados. O Programa tem três propósitos primários: (1) construir e/ou consolidar capacitação interna em sensoriamento remoto por radar nos países participantes; (2) avaliar o potencial e demonstrar a aplicabilidade do RADARS AT-1 em várias aplicações em áreas prioritárias de cada país voltadas ao levantamento e monitoramento de recursos naturais renováveis e não-renováveis e estudos ambientais; (3) ampliar o estabelecimento de vínculos e intercâmbio entre instituições públicas e privadas canadenses e parceiros similares na América Latina. No total, 116 propostas foram submetidas no Programa GloeSAR-2, sendo 65 aprovadas com a aquisição de imagens especialmente programadas aos objetivos de cada projeto de pesquisa e aplicação.

O GlobeSAR-2 NO BRASIL $\mathrm{Na}$ discussão sobre a concepção do Programa no Brasil, ficou acordado que a participação das universidades seria fundamental para $o$ sucesso de uma iniciativa que buscava consolidar a capacitação nacional no campo de sensoriamento remoto nas microondas. Neste sentido, houve um grande esforço de divulgação dos objetivos do Programa nas principais universidades do país, que de alguma forma, contassem com um mínimo de massa crítica em sensoriamento remoto para propor um projeto de pesquisa, receber treinamento e capacitação, desenvolver a investigação, e tornar-se um polo disseminador da tecnologia, regional e nacionalmente. Também ficou acertado, que a participação no Programa ocorreria a partir da submissão de propostas de projetos de pesquisa, que seriam julgadas em bases competitivas, e aprovadas por critérios de mérito, por equipe externa (CCRS e RSI). Desta forma, atendendo à solicitação do INPE, 28 propostas de projetos foram submetidas ao Programa em Março de 1996 e os resultados comunicados aos participantes em Outubro de 1996. Deste total, 10 propostas foram recomendadas à CIDA, como projetos aprovados para aquisição de cenas do RADARS AT-1 (Tabela 2). Das propostas aprovadas para recebimento de

Tabela 2. Propostas aprovadas no GlobeSAR-2 para recebi-

\begin{tabular}{|c|c|c|c|c|}
\hline Pinpouta (Na-t & Titewlo & 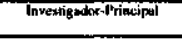 & Jififi & $\begin{array}{l}\text { Tamacconat } \\
\text { RADARSAT }\end{array}$ \\
\hline 1 & 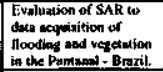 & 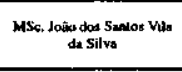 & 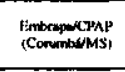 & $\begin{array}{l}\text { Aniline Antientsl } \\
\text { OA cenass }\end{array}$ \\
\hline 2 & 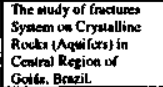 & $\begin{array}{l}\text { Da, Paulo Ruberts } \\
\text { Motetsis }\end{array}$ & 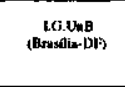 & Oof cenass \\
\hline$t$ & 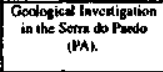 & 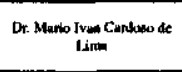 & 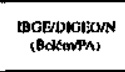 & crostagin \\
\hline 12 & 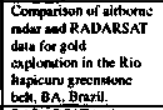 & 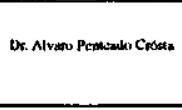 & $\begin{array}{l}\text { I.G. UNHCAMAP } \\
\text { (Campinan'SP) }\end{array}$ & lisakengid \\
\hline is & 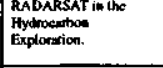 & Ix. Ruimusato Almeida & 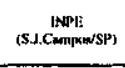 & Gontegin \\
\hline 20 & 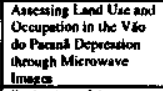 & Dr. Edhoos Sanus & 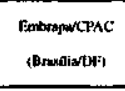 & 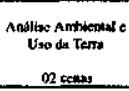 \\
\hline 21 & 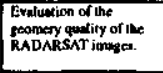 & 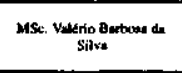 & 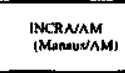 & 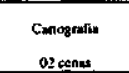 \\
\hline 22 & 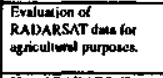 & 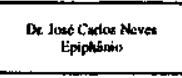 & 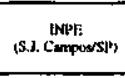 & 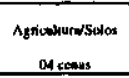 \\
\hline 35 & 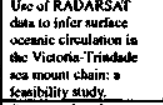 & 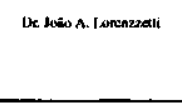 & 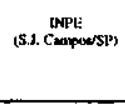 & 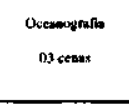 \\
\hline 20 & 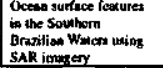 & 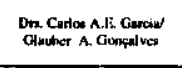 & $\begin{array}{l}\text { D.FnRG } \\
\text { (Rio GrandL/RCS) }\end{array}$ & 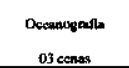 \\
\hline
\end{tabular}

mento de cenas RADARSAT-1. dados RADARSAT-1, 04 são de Geologia, 02 de Oceanografia, 02 de Análise Ambiental, 01 de Cartografia e 01 de Agricultura/Solos. Além de suporte às diferentes reuniões do Programa, fundos estão disponíveis para trabalho de campo dos projetos aprovados e licenças dos softwares de tratamento de imagens de radar (PCI e Earth-View) foram cedidos aos participantes destas propostas.

O COMPONENTE EDUCACIONAL DO PROGRAMA A grande participação das universidades no Anúncio de Oportunidades do Programa levou a que a coordenação do GlobeSAR, na impossibilidade de atender às solicitações de imagens do RADARSAT-1, devido às limitações orçamentarias, optasse por incluir em um segundo conjunto, propostas, que pela excelência da qualidade, receberão um pacote educacional completo de radar (imagens de aplicacões do RADARSAT em CD-ROM, transparências, apostilas, etc..) além de licenças dos softwares PCI e EarthView. Um representante de cada universidade atendeu ao Workshop I realizado no INPE em Junho/Julho de 1997 com fundos do Programa. Na tabela 3 são listadas as propostas das universidades incluídas neste segundo grupo. Um aspecto importante deste componente universitário é a alocação de fundos para intercâmbios entre docentes e alunos de universidades brasileiras e canadenses. O Programa apoiará visitas de curta duração (l a 3 meses), entre equipes dos dois países, para desenvolver pesquisa e capacitação conjunta dentro do escopo do Programa.

\section{PRINCIPAIS EVENTOS DO GLOBESAR-2 No} planejamento do GlobeSAR-2 foram previstos um Workshop Inicial, dois Seminários Nacionais e dois Simpósios Internacionais. $\mathrm{O}$ Workshop Inicial foi realizado no período de 25 Junho - 04 Julho de 1997, no INPE (São José dos Campos, SP). Além de um curso avançado sobre SAR (teoria e aplicações), ministrado por especialistas do CCRS/INPE, dois treinamentos independentes sobre tratamento digital de imagens SAR , com os pacotes Easi-Pace e Earth View, foram oferecidos por equipes da PCI e Atlantis Scientific, respectivamente, a todos os participantes com propostas aprovadas no Programa (projetos aprovados com cenas e grupo universitário). Em Fevereiro de 1998 (16-17) foi realizado no INPE o Seminário Nacional I do GlobeSAR-2, com a apresentação dos primeiros resultados dos projetos de pesquisa do Programa. Em Abril está previsto o First GlobeSAR-2 International Symposium (21-24 de Abril, Cartagena, Colômbia). Ainda durante a realização do IX Simpósio Brasileiro de Sensoriamento Remoto (11-18 de Setembro, Santos,) está previsto um Workshop sobre o Programa. Para 1999 estão previstos o Seminário Nacional II do GlobeS AR-2 (Fevereiro, INPE) e Final GlobeSAR International Symposium (Maio, local ainda não definido). O Programa GlobeSAR-2 terá seu término no final de 1999. A figura 2 mostra a primeira cena RADARSAT-1 ScanSAR do Brasil, , adquirida em 16 de Novembro de 1996 na Província Mineral de Carajás, Pará.

Tabela 3. Propostas aprovadas no Grupo Universidades do Programa GlobeSAR-2

\begin{tabular}{|c|c|c|c|}
\hline Pioposta (No.) & Investigador-princjpal & Filiaçá & Arca de aplicaḉ \\
\hline 5,6 & Dr. MaÂmar El-Robsini & C.G.ЛFPA & Ceologia \\
\hline 13 & Dr. J. Batista Sena Costa & C.G.JUFPA & Geotogia \\
\hline 9 & Dr, Jorgc S. Bettencourt & L.ONSP & Geologia \\
\hline 8 & Dr. Toodoro L.R. Almeida & 1.G.MSPP & Geodogia \\
\hline 14 & Dr. E. F. Jardiun de $S \mathbf{S}$ & D.G.AFRN & Gcologia \\
\hline 10 & Dr. Clovis C. Camrario & D.G.JUFRGS & Geologia \\
\hline iI & Dr. Alhenino S. Carvalho & D.G.M.F.AM/AM & Goologia \\
\hline 15 & Dr. Albeno Pio Fiori & D.G $\Omega$ FPR/PR & Geologia \\
\hline $1 \overline{8}$ & Dra Celina Foresti & I.B JUNESP/S.P. & Uso do Solo Urbano \\
\hline 27 & Dr, Gilhero J. Garcia & CEAPLANUNESPSS.P. & Análise Ambiental \\
\hline 19 & Dra. Sandea M.F. da Costa & I.P.D/UNIVAPIS.P. & Uso do Solo Uirbano \\
\hline 23 & D..A. P erez Filho & UNICAMPSS.P. & Análise Ambiental \\
\hline 28 & Dra. Rosana M. Rodrizues & U. Vale do ItajdidSC & Geofogia Marinha \\
\hline
\end{tabular}




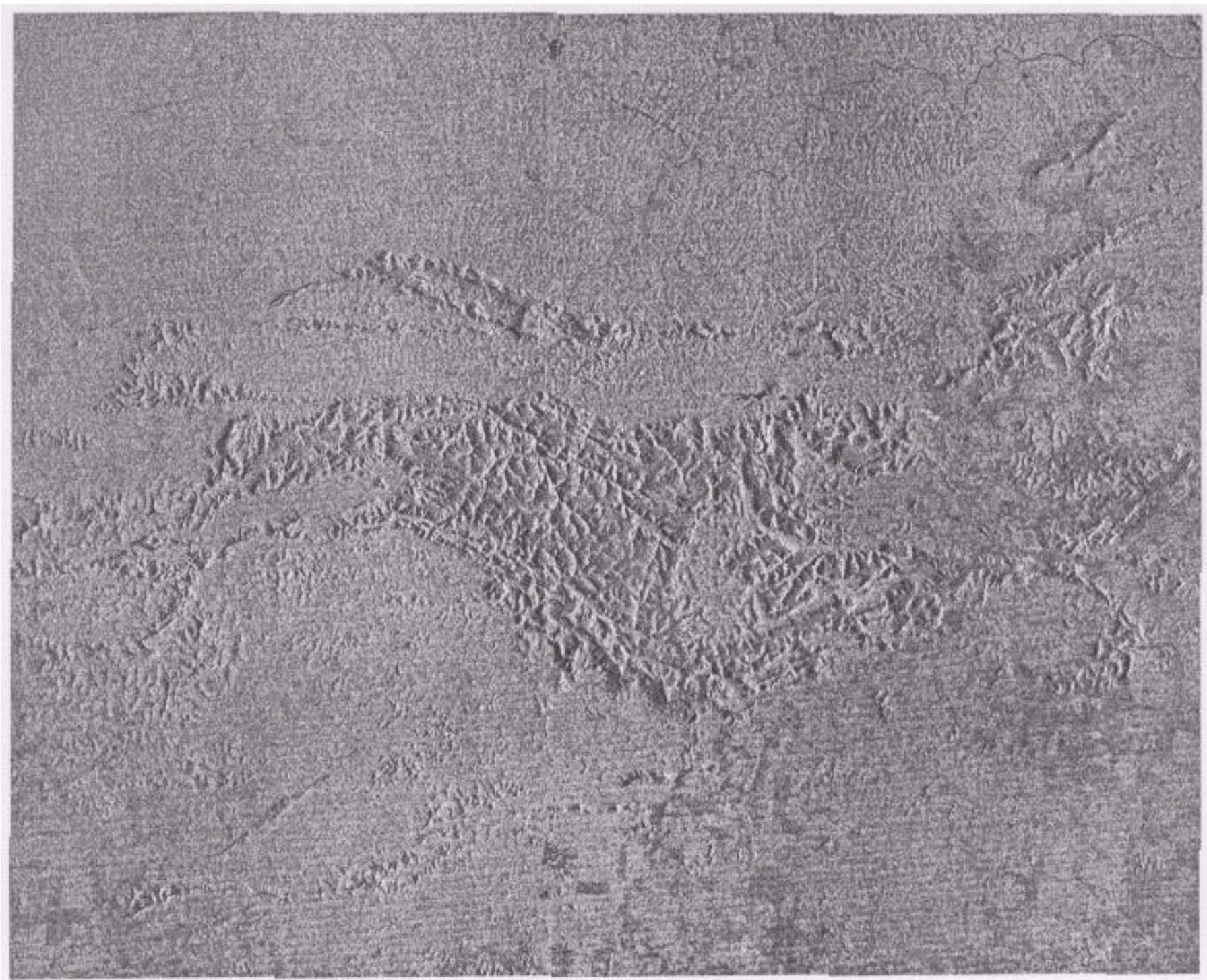

Figura 2. Imagem RADARSAT-l ScanSAR Narrow A, adquirida em 16 de Novembro de 1996 da Província Mineral de Carajás, Pará. A área aproximada da figura é de $250 \times 190 \mathrm{Km}$ (fonte: RSI/CSA, ADRO \# 364).

\section{Referências}

Brisco, B.; Manore, M.J.; Brown, R.J.; D'Iorio, M.A.; Campbell, F.H.A. 1997. GlobeSAR-2 Program and RADARSAT Applications Review. In: Natural Resources Canada, Ottawa, Canada, International Symposium: Geomatics in the Era of RADARSAT, CD-ROM dos Proceedings.

Brown, R.J.; Brisco, B.; D'Iorio, M.A.; Prevost, C.; Ryerson, R.A.; Singhroy, V.H. 1996. RADARSAT Applications: Review of GlobeSAR Program. Canadian Journal of Remate Sensing, 22 (4): 404-419.

ES A (European Space Agency) 1993. South American Radar Experiment. In: ESA-Workshop WPP-76, Paris, Proceedings, 246 p.

Paradella, W.R. 1996a. Imagens de Radar: Fundamentação e Experiências com o SAR na Amazônia. FATOR G/5, 14 (Jun/Jul): 40-43.

Paradella, W.R. 1996b. O RADARSAT-1 e as Perspectivas de Aplicações em Geologia. In: SBG, Cong. Bras. Geol., 37, Salvador, Anais, 7: 105-109.

Manuscrito NB-15

Recebido em 20 de Fevereiro de 1998 Revisão dos autores em 02 de Março de 1998 Revisão aceita em 05 de Março de 1998 